\title{
Regularity of degenerate Hessian equations
}

\author{
Sławomir Dinew ${ }^{1} \cdot$ Szymon Pliś ${ }^{2} \cdot{\text { Xiangwen } \text { Zhang }^{3}}^{3}$
}

Received: 9 June 2018 / Accepted: 30 May 2019 / Published online: 17 July 2019

(c) The Author(s) 2019

\begin{abstract}
We show a second order a priori estimate for solutions to the complex $k$-Hessian equation on a compact Kähler manifold provided the $(k-1)$ st root of the right hand side is $\mathcal{C}^{1,1}$. This improves an estimate of Hou-Ma-Wu (Math Res Lett 17:547-561, 2010). An example is provided to show that the exponent is sharp.
\end{abstract}

Mathematics Subject Classification Primary 35J60; Secondary 35B45

\section{Introduction}

Geometrically motivated complex fully nonlinear elliptic partial differential equations have received a lot of attention recently (see [16-18,20,21] which is by far an incomplete list of recent important contributions). The solvability of such equations is usually studied through the continuity method and boils down to establishing a priori estimates just as in the classical approach of Yau [25].

In general the considered problems are reducible to a scalar equation satisfied by a real valued function $u$ defined of a compact complex manifold $X$ equipped with a fixed Hermitian form $\omega$. Quite often additional assumptions such as kählerness of $\omega$ are imposed and then the real $(1,1)$-form $\omega+i \partial \bar{\partial} u$ is the geometric object with the desired properties. Arguably the most natural geometric assumption is that $\omega+i \partial \bar{\partial} u$ defines a metric i.e. it is positive

Communicated by O. Savin.

The first and second named authors were supported by the NCN Grant 2013/08/A/ST1/00312. The third named author was supported by the Simons Collaboration Grant-523313.

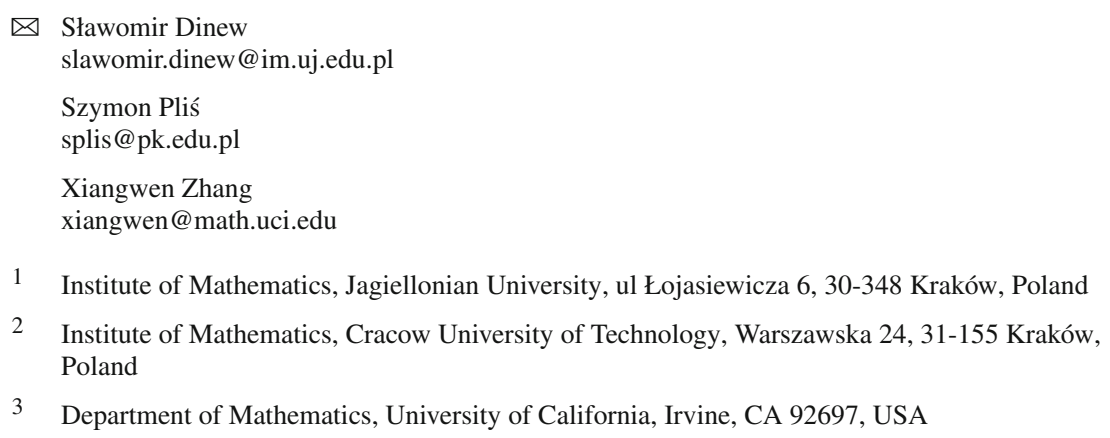


definite. However, it often happens that the very nature of the nonlinearity imposes more general admissibility conditions (see for example $[9,10,16,20]$ ). This lack of positivity usually contributes significantly to the technical difficulty of the estimates.

In this note we deal with the complex Hessian equations on a compact Kähler manifold $(X, \omega)$ with $\operatorname{dim}_{\mathbb{C}} X=n$. These interpolate between the Laplace equation (in the case $k=1$ ) and the Monge-Ampère equation in the case $k=n$. They are defined by

$$
(\omega+i \partial \bar{\partial} u)^{k} \wedge \omega^{n-k}=f \omega^{n},
$$

where the given nonnegative function $f$ satisfies the necessary compatibility condition $\int_{X} \omega^{n}=\int_{X} f \omega^{n}$.

For smooth $u$ the admissibility condition imposed of the class of solutions $u$ is that

$$
(\omega+i \partial \bar{\partial} u)^{j} \wedge \omega^{n-j} \geq 0, \quad j=1,2, \ldots, k .
$$

We denote the class of such functions by $\mathcal{S H}_{k}(X, \omega)$. Note also that adding a constant to a solution $u$ doesn't change the Eq. (1.1), thus we normalize the solutions by imposing the condition $\int_{X} u \omega^{n}=0$. The solvability of Eq. (1.1) was established for smooth strictly positive right hand side data $f$ satisfying the compatibility condition through the works of Hou-Ma-Wu [10] who proved the uniform and second order a priori estimates and the first named author and Kołodziej [6] who obtained the missing gradient estimate by an indirect blow-up argument.

Having the existence of smooth solutions for smooth strictly positive data it is natural to address the regularity theory in the degenerate cases. A situation of special interest is when the right hand side function is allowed to vanish. Such a scenario, reminiscent of failure of strict ellipticity in linear PDEs, as a rule implies the occurrence of singular solutions. In view of the classical theory in the Monge-Ampère case (see $[3,7])$ the maximum one can expect in this setting is $\mathcal{C}^{1,1}$ regularity.

A natural question appears then about optimal conditions implying that $u \in \mathcal{C}^{1,1}$. Note that in [10] the authors have proven that the complex Hessian is controlled by the gradient of $u$ provided the $\mathcal{C}^{2}$ norm of $f^{1 / k}$ is under control. This may hold even if $f$ vanishes somewhere, that is, we deal with the degenerate equation. The estimate in [10] left the problem whether the exponent $1 / k$ is the optimal one. We will show that one can further improve it to $1 /(k-1)$ if $k \geq 2$ as one expects for the real case (for $k=1$ we have the Poisson equation whose regularity theory is classical). Our main result is the following:

Theorem 1.1 Let $f \geq 0$ be a function on compact Kähler manifold $(X, \omega)$ satisfying $\int_{X} f \omega^{n}=\int_{X} \omega^{n}$. Assume that $f^{1 /(k-1)} \in \mathcal{C}^{1,1}$. Then the solution $u$ to Eq. (1.1) admits an a priori estimate

$$
\sup _{X}\|i \partial \bar{\partial} u\| \leq C\left(1+\|D u\|^{2}\right)
$$

for some uniform constant $C$ dependents only on $n, k, X,\left\|f^{1 /(k-1)}\right\|_{\mathcal{C}^{1,1}}$, the oscillation $o^{o s} c_{X} u$ of $u$ and the lower bound on the bisectional curvature of $\omega$.

Coupling this estimate with the main result from [6] one can prove that the solution $u$ has bounded Laplacian and thus belongs to the weak $\mathcal{C}^{1,1}$ space. The proof of the above estimate relies heavily on the argument of $\mathrm{Hou}-\mathrm{Ma}-\mathrm{Wu}$ [10]. The main importance of our improvement is that the obtained exponent is optimal as an example constructed in the note shows.

The complex Hessian equations were first considered in the case of domains in $\mathbb{C}^{n}$, where the equation takes the form 


$$
(i \partial \bar{\partial} u)^{k} \wedge \beta^{n-k}=f \beta^{n}
$$

with $\beta=d d^{c}|z|^{2}$ denoting the standard Hermitian $(1,1)$ form in $\mathbb{C}^{n}$. The corresponding Dirichlet problem was studied by Li [14] and Błocki [2]. In particular, the nondegenerate Dirichlet problem in a strictly k-pseudoconvex domain admits a unique smooth solution provided $f$ and the boundary data are smooth and $f$ is uniformly positive. Again it is interesting to study $\mathcal{C}^{1,1}$ regularity in the case when $f$ vanishes or decreases to zero at the boundary. It has to be emphasized that the occurrence of a boundary makes things substantially harder and the regularity theory is far from complete. When $k=n$, that is for the complex MongeAmpère case, some regularity results were obtained by Krylov $[12,13]$ under the assumption that $f \geq 0$ and $f^{1 / k} \in \mathcal{C}^{1,1}$.

The complex Hessian equation is itself modelled on its real counterpart

$$
S_{k}\left(D^{2} u\right)=f
$$

with $S_{k}(A)$ denoting the sum of all main $k \times k$-minors of the matrix $A$. The real Hessian equation is much better understood and we refer to [24] for an excellent survey regarding the corresponding regularity theory. In particular in the real setting the following analogue of the Hou-Ma-Wu [10] estimate was established by Ivochkina-Trudinger-Wang in [11].

Theorem 1.2 [11] Let $U \subset \mathbb{R}^{n}$ be a strictly $k$-convex domain with $C^{4}$ boundary. Suppose that the admissible function $v$ satisfies the problem

$$
\begin{cases}S_{k}\left(D^{2} v\right)=f & \text { in } U \\ v=\varphi & \text { in } \partial U,\end{cases}
$$

where we assume that $\varphi \in \mathcal{C}^{4}(\partial U)$ and $f^{1 / k} \in \mathcal{C}^{2}(\bar{U})$. Then $v \in \mathcal{C}^{1,1}(\bar{U})$ with $\mathcal{C}^{2}$ norm bounded by an estimable constant dependent on $f, \varphi, k, n$ and $U$.

Again it is unknown whether the exponent $1 / k$ is optimal here. It has attracted much attention to establish the above theorem with the exponent $1 / k$ being replaced by $1 /(k-1)$. More recently, the above theorem was proved under a weaker condition on $f$ in [22], but the optimal one seems still missing. On the bright side the optimality problem has been settled in the extremal case $k=n$, i.e. when we deal with the real Monge-Ampère equation. By a result of Guan-Trudinger-Wang [8] the optimal exponent yielding $\mathcal{C}^{1,1}$ solutions is $1 /(n-1)$ for domains in $\mathbb{R}^{n}$. Sharpness of this bound follows from an example of Wang [23]. This example has been generalized for the complex Monge-Ampère equation by the second named author in [19].

In the case of general Hessian equations the current state of affairs is as follows: it was stated in [11] that an example analogous to the one in [23] suggests that the exponent $1 /(k-1)$ is optimal for the real $k$-Hessian equation. As no proof of this was provided we take the opportunity to present the relevant example (as well as its complex and compact manifold counterparts) in detail, since in our opinion the arguments used in the proof have to be slightly different than the approach of Wang [23]. In particular, we have

Proposition 1.3 For every $\varepsilon>0$, there exists a non-negative function $f$ in the unit ball in $\mathbb{R}^{n}$ (respectively, $\mathbb{P}^{n-1} \times \mathbb{P}^{1}$ or the unit ball in $\mathbb{C}^{n}$ ) such that $f^{1 /(k-1)+\varepsilon} \in \mathcal{C}^{1,1}$, but the solution to the $k$-Hessian equation with $f$ as a right hand side is not $\mathcal{C}^{1,1}$.

The examples living on $\mathbb{P}^{n-1} \times \mathbb{P}^{1}$ equipped with the Fubini-Study product metric yield in particular a regularity threshold $1 /(k-1)$ for the exponent of $f$. This shows that our main result (Theorem 1.1) is optimal. We also take the opportunity to investigate the regularity of 
the example given in Proposition 1.3 under various weaker assumptions on the right hand side (see Example 4.6 in Sect. 4.2). More precisely, we provide some examples to indicate what might be the best possible regularity of the admissible solutions for equation

$$
(\omega+i \partial \bar{\partial} u)^{k} \wedge \omega^{n-k}=f(z) \omega^{n},
$$

on a compact Kähler manifold $(X, \omega)$ with $0 \leq f \in L^{p}$ (or $\mathcal{C}^{0, \delta}$ ) satisfying $\int_{X} f \omega^{n}=\int_{X} \omega$. We believe that at least in some cases the obtained examples are sharp.

\section{Preliminaries}

Below we gather the definitions and facts that will be used in the proofs later on. We refer to the survey article [24] for the basics of the theory of Hessian equations. We start with some relevant notions from linear algebra. Consider the set $\mathcal{M}_{n}(\mathbb{R})$ (respectively: $\mathcal{M}_{n}(\mathbb{C})$ ) of all symmetric (respectively Hermitian symmetric) $n \times n$ matrices. Let $\lambda(M)=\left(\lambda_{1}, \lambda_{2}, \ldots, \lambda_{n}\right)$ be the eigenvalues of a matrix $M$ arranged in decreasing order and let

$$
S_{k}(M)=S_{k}(\lambda(M))=\sum_{0<j_{1}<\cdots<j_{k} \leq n} \lambda_{j_{1}} \lambda_{j_{2}} \ldots \lambda_{j_{k}}
$$

be the $k$ th elementary symmetric polynomial applied to the vector $\lambda(M)$. Analogously we define $\sigma_{k}(M)$ if $M$ is Hermitian. Then one can define the positive cones $\Gamma_{m}$ as follows

$$
\Gamma_{m}=\left\{\lambda \in \mathbb{R}^{n} \mid S_{1}(\lambda)>0, \ldots, S_{m}(\lambda)>0\right\} .
$$

Note that the definition of $\Gamma_{m}$ is nonlinear if $m>1$.

Let now $V=\left(v_{\bar{k} j}\right)$ be a fixed positive definite Hermitian matrix and $\lambda_{i}(T)$ be the eigenvalues of a Hermitian matrix $T=\left(\tau_{\bar{k} j}\right)$ with respect to $V$. We can define analogously $\sigma_{k, V}(T)$. In the language of differential forms if $\tau=i \tau_{\bar{k} j} d z^{j} \wedge d \bar{z}^{k}, v=i v_{\bar{k} j} d z^{j} \wedge d \bar{z}^{k}$ then $\sigma_{k, V}(T)$ is (up to a multiplicative universal constant) equal to the coefficient of the top-degree form $\tau^{k} \wedge v^{n-k}$. We can also analogously define the sets $\Gamma_{k}(V)$. Below we list the properties of these cones that will be used later on:

1. (Maclaurin's inequality I) If $\lambda \in \Gamma_{m}$ then $\left(\frac{S_{j}}{\left(\begin{array}{c}n \\ j\end{array}\right)}\right)^{\frac{1}{j}} \geq\left(\frac{S_{i}}{\left(\begin{array}{c}n \\ i\end{array}\right)}\right)^{\frac{1}{i}}$ for $1 \leq j \leq i \leq m$. The same inequality holds for the operators $\sigma_{k}$;

2. (Maclaurin's inequality II) There is a universal constant $c(n, m)$, dependent only on $n$ and $m$, such that $\sigma_{m-1}(\lambda) \geq c(n, m) \sigma_{m}(\lambda)^{\frac{m-2}{m-1}} \sigma_{1}(\lambda)^{\frac{1}{m-1}}$ for any $\lambda \in \Gamma_{m} ;$

3. $\Gamma_{m}$ is a convex cone for any $m$ and the function $\sigma_{m}^{\frac{1}{m}}$ as well as $\log \left(\sigma_{m}\right)$ are concave when restricted to $\Gamma_{m}$;

4. (Gårding's inequality) Let $\sigma_{k}(\lambda \mid i):=\frac{\partial \sigma_{k+1}}{\partial \lambda_{i}}(\lambda)$. Then for any $\lambda, \mu \in \Gamma_{m}$

$$
\sum_{i=1}^{n} \mu_{i} \sigma_{m-1}(\lambda \mid i) \geq m \sigma_{m}(\mu)^{\frac{1}{m}} \sigma_{m}(\lambda)^{\frac{m-1}{m}} .
$$

5. $\sigma_{m-1}(\lambda \mid i j)=\frac{\sigma_{m}(\lambda \mid i)-\sigma_{m}(\lambda \mid j)}{\lambda_{j}-\lambda_{i}}$ for all $i \neq j$.

We refer to [24] for further properties of these cones.

Recall that a smooth function $v$ living on a domain $U \subset \mathbb{R}^{n}$ is called $k$-convex for some natural $1 \leq k \leq n$ if

$$
S_{j}\left(D^{2} v(x)\right) \geq 0, \quad j=1, \ldots, k
$$


with $D^{2} v(x)$ denoting the Hessian matrix of $v$ at $x$ and $S_{j}(A)$ is the sum of all main $j \times j$ minors of the $n \times n$ matrix $A$. Analogously a function $u$ living on a domain $\Omega \subset \mathbb{C}^{n}$ is called $k$-subharmonic for some natural $1 \leq k \leq n$ if

$$
\sigma_{j}(i \partial \bar{\partial} u(z)) \geq 0, \quad j=1, \ldots, k
$$

with $\sigma_{j}(B)$ denoting again the sum of the main $j \times j$ minors of a Hermitian symmetric matrix $B$. In the complex setting one can alternatively use the language of differential forms to define the $\sigma_{k}$ operator as

$$
\sigma_{k}(i \partial \bar{\partial} u) \beta^{n}=\left(\begin{array}{l}
n \\
k
\end{array}\right)(i \partial \bar{\partial} u)^{k} \wedge \beta^{n-k}
$$

with $\beta:=d d^{c}|z|^{2}$ denoting the standard Hermitian $(1,1)$-form in $\mathbb{C}^{n}$.

These are the local real and complex versions of the functions belonging to $\mathcal{S H}_{k}(X, \omega)$ defined in the introduction. In each of these settings one can define singular $k$-convex (respectively $k$-subharmonic) functions locally as decreasing limits of smooth ones. The basic fact from the associated nonlinear potential theories (see [24] for the real case and [2,5] for the complex one) is that the operators $S_{k}$ (respectively $\sigma_{k}$ ) can still be properly defined as nonnegative measures for singular bounded $k$-convex ( $k$-subharmonic) functions.

The following theorems, known as comparison principles are basic in the potential theory of $k$-subharmonic and $k$-convex functions (see [5,24]).

Theorem 2.1 [24] If $u, w$ are two bounded $k$-convex functions in a domain $U \subset \mathbb{R}^{n}$, such that $\liminf _{x \rightarrow \partial \Omega}(u-w)(x) \geq 0$. If moreover

$$
S_{k}\left(D^{2} w\right) \geq S_{k}\left(D^{2} u\right)
$$

as measures then $u \geq w$ in $U$.

Theorem 2.2 [5] If $u, w$ are two bounded $k$-subharmonic functions in a domain $\Omega \subset \mathbb{C}^{n}$, such that $\lim _{\inf _{z \rightarrow \partial \Omega}}(u-w)(z) \geq 0$. If moreover

$$
\sigma_{k}(i \partial \bar{\partial} w) \geq \sigma_{k}(i \partial \bar{\partial} u)
$$

as measures then $u \geq w$ in $\Omega$.

As a corollary one immediately obtains the uniqueness of bounded solutions for the corresponding Dirichlet problems. The uniqueness of normalized bounded solutions from $\mathcal{S H}_{k}(X, \omega)$ is also true (see [4]). The corresponding comparison principle (see [6]) reads as follows:

Theorem 2.3 Let $\varphi, \psi \in \mathcal{S H}_{k}(X, \omega)$ be bounded. Then

$$
\int_{\{\varphi<\psi\}}(\omega+i \partial \bar{\partial} \psi)^{k} \wedge \omega^{n-k} \leq \int_{\{\varphi<\psi\}}(\omega+i \partial \bar{\partial} \varphi)^{k} \wedge \omega^{n-k} .
$$

Finally we shall need an elementary calculus lemma whose proof can be found in [1]:

Lemma 2.4 If $\psi \in \mathcal{C}^{1,1}(\bar{\Omega})$ is a nonnegative function. Then $\sqrt{\psi}$ is locally Lipschitz in $\Omega$. For almost every $x \in \Omega$ we have

$$
|D \sqrt{\psi}(x)| \leq \max \left\{\frac{|D \psi(x)|}{2 \operatorname{dist}(x, \partial \Omega)}, \frac{1+\sup _{\Omega} \lambda_{\max }\left[D^{2} \psi\right]}{2}\right\},
$$

where $\lambda_{\max }\left[D^{2} \psi\right]$ denotes the maximum eigenvalue of the real Hessian of $\psi$. 
Working in charts on a compact Kähler manifold one easily gets the following corollary of the lemma above:

Corollary 2.5 Let $f \geq 0$ be a function on a compact Kähler manifold $(X, \omega)$ such that $f^{1 /(k-1)} \in \mathcal{C}^{1,1}(X)$. Then

$$
\left\|\nabla f^{1 /(k-1)}(z)\right\|^{2} \leq C\left\|f^{1 /(k-1)}(z)\right\|
$$

for some constant $C$ dependent on $X, \omega$ and the $\mathcal{C}^{1,1}$ norm of $f^{1 /(k-1)}$. In particular for any unitary vector $\eta$ one has

$$
\partial_{\eta} \partial_{\bar{\eta}} \log f=(k-1)\left(\frac{\partial_{\eta} \partial_{\bar{\eta}} f^{1 /(k-1)}}{f^{1 /(k-1)}}-\frac{\left|\partial_{\eta} f^{1 /(k-1)}\right|^{2}}{f^{2 /(k-1)}}\right) \geq-\frac{\tilde{C}}{f^{1 /(k-1)}}
$$

for some constant $\tilde{C}$ dependent on $X, \omega$ and the $\mathcal{C}^{1,1}$ norm of $f^{1 /(k-1)}$.

Proof Pick a point $z \in X$ and a chart centered around $z$ containing a ball of some fixed radius $r$ (dependent only on $X$ and $\omega$ ). Then we apply the lemma for $\psi=f^{1 /(k-1)}$ in the coordinate ball centered at $z$ with radius $r$ to get the statement.

Remark 2.6 In the corollary it is crucial that the manifold has no boundary. As observed in [1] the function $\psi(t)=t$ on $(0,1)$ shows that it is in general impossible to control $|D \psi|^{2}$ by $\psi$ globally in the presence of boundary.

Notation Throughout the paper, $(X, \omega)$ will denote a compact Kähler manifold, $\Omega$ will be a domain in $\mathbb{C}^{n}$ and $U$ will be a domain in $\mathbb{R}^{n}$ for some $n \geq 2$. The constant $C_{0}$ denotes the lower bound for the bisectional curvature associated to $\omega$ i.e.

$$
C_{0}:=\sup _{x \in M}\left|\inf _{\eta, \zeta} R_{\eta \bar{\eta} \zeta \bar{\zeta}}\right|
$$

with $\zeta, \eta$ varying among the unit vectors in $T_{x} X$. Other constants dependent only on the pertinent quantities will be denoted by $C, C_{i}$ or $c_{i}$. We shall refer to these as constants under control.

\section{The main estimate}

This section is devoted to the proof of the following a priori estimate:

Theorem 3.1 Let $u \in \mathcal{S H}_{k}(X, \omega)$ be a $\mathcal{C}^{4}(X)$ function solving the problem

$$
\left\{\begin{array}{l}
(\omega+i \partial \bar{\partial} u)^{k} \wedge \omega^{n-k}=f \omega^{n} \\
\int_{X} u \omega^{n}=0
\end{array}\right.
$$

where the nonnegative function $f$ satisfies the compatibility assumption $\int_{M} f \omega^{n}=\int_{M} \omega^{n}$. Suppose that $\|f\|_{\mathcal{C}^{0}} \leq B,\left\|f^{1 /(k-1)}\right\|_{\mathcal{C}^{1}} \leq B$ and $\left\|f^{1 /(k-1)}\right\|_{\mathcal{C}^{2}} \leq B$. Then

$$
\sup _{M}\|i \partial \bar{\partial} u\|_{\omega} \leq C\left(\sup _{M}\|\nabla u\|^{2}+1\right)
$$

for some constant $C$ dependent on $C_{0}, B, \omega, n$ and $k$. 
Using the above $\mathcal{C}^{2}$ estimate, one can repeat the blow-up argument from [6] to deduce an indirect gradient bound for $u$. Coupling this information with (3.2), we get the following result:

Theorem 3.2 If $u \in \mathcal{S H}_{k}(X, \omega)$ solves the problem (3.1) with the assumption $f^{1 /(k-1)} \in$ $\mathcal{C}^{1,1}$, then $u$ belongs to the weak $\mathcal{C}^{1,1}$ space, i.e. the Laplacian of $u$ is bounded.

Proof of Theorem 3.2 The argument can be found in [1]. We provide the details for the sake of completeness.

Given any $f$ as in the statement there is a family $f_{\epsilon}, \epsilon \in(0,1)$ of smooth strictly positive functions uniformly convergent as $\epsilon \searrow 0$ to $f$ such that additionally $f_{\epsilon}^{1 /(k-1)}$ tends to $f^{1 /(k-1)}$ in $\mathcal{C}^{1,1}$ norm (one way to produce such a family is to use a convolution in local charts coupled with a partition of unity, see [1] for the details). Let also $C_{\epsilon}$ be a positive constant such that

$$
\int_{M} C_{\epsilon} f_{\epsilon} \omega^{n}=\int_{M} f \omega^{n}=\int_{M} \omega^{n}
$$

It follows that $\lim _{\epsilon \rightarrow 0} C_{\epsilon}=1$. Furthermore we can assume that

$$
\left\|\left(C_{\epsilon} f_{\epsilon}\right)^{1 /(k-1)}\right\|_{\mathcal{C}^{2}} \leq 2\left\|f^{1 /(k-1)}\right\|_{\mathcal{C}^{2}} .
$$

Hence the solutions $u_{\epsilon} \in \mathcal{S H}_{k}(X, \omega)$ to the problem

$$
\left\{\begin{array}{l}
\left(\omega+i \partial \bar{\partial} u_{\epsilon}\right)^{k} \wedge \omega^{n-k}=C_{\epsilon} f_{\epsilon} \omega^{n} \\
\int_{X} u_{\epsilon} \omega^{n}=0
\end{array}\right.
$$

(which are smooth by the Calabi-Yau type theorem from [6]) converge in $L^{1}(X, \omega)$ to $u$ (see Corollary 4.2 in [15]).

On the other hand we have as an application of Theorem 3.1 the bound $\Delta_{\omega} u_{\epsilon} \leq C$ for a constant $C$ dependent only on $\left\|f^{1 /(k-1)}\right\|_{\mathcal{C}^{2}}, n, k$ and the lower bound of the bisectional curvature. In particular the bound does not depend on $\epsilon$ and hence passing to the limit we obtain $\Delta_{\omega} u \leq C$ which implies the claimed result.

Now we proceed to the proof of the main a priori estimate:

Proof of Theorem 3.1 We will work, just as in [1] under the assumption that $f>0$ (for example using the approximate problems (3.3)) and we will obtain an estimate independent of $\inf _{X} f$. This is done in order to avoid confusion as we shall divide by $f$ in the argument. Then, if needed, one can repeat the final part of the argument in the proof of Theorem 3.2 to drop the assumption $f>0$. Our proof will follow closely the argument in [10].

Given a point $x \in M$ we consider a fixed local coordinate system $\left(z_{1}, \ldots, z_{n}\right)$ centered at $x$. By re-choosing the coordinates if necessary, one can assume that the form $\omega=i g_{\bar{k} j} d z^{j} \wedge d \bar{z}^{k}$ is diagonal at $x$. We follow the notation in [10] and use the covariant derivatives with respect to the background Kähler metric $\omega$ to do the calculation. In particular for any function $h$ defined near $x$ let $h_{i}=\nabla_{\partial / \partial z^{i}} h, h_{i \bar{j}}=\nabla_{\partial / \partial \bar{z}^{j}} \nabla_{\partial / \partial z^{i}} h$, etc.

As in [10], we consider the quantity

$$
\tilde{G}(z, \xi):=\log \left(1+u_{i} \bar{j}^{i} \bar{\xi}^{j}\right)+\varphi\left(|\nabla u|^{2}\right)+\psi(u)
$$

defined for any $z \in X$ and any unit vector $\xi \in T_{z}^{1,0} X$. The relevant quantities are defined as follows:

$$
\varphi(t):=-\frac{1}{2} \log \left(1-\frac{t}{2 K}\right) \quad \text { with } \quad K:=\sup _{M}|\nabla u|^{2}+1 ;
$$


and

$$
\psi(t):=-A \log \left(1+\frac{t}{2 L}\right) \quad \text { with } \quad L:=\sup _{M}|u|+1, \quad A=3 L\left(2 C_{0}+1\right) .
$$

The properties of $\varphi$ and $\psi$ that we shall use are listed below:

$$
\begin{gathered}
\frac{1}{2} \log 2 \geq \varphi\left(|\nabla u|^{2}\right) \geq 0, \quad \frac{1}{2 K} \geq \varphi^{\prime}\left(|\nabla u|^{2}\right) \geq \frac{1}{4 K}>0, \\
\varphi^{\prime \prime}\left(|\nabla u|^{2}\right)=2\left[\varphi^{\prime}\left(|\nabla u|^{2}\right)\right]^{2}>0
\end{gathered}
$$

and

$$
\begin{aligned}
& A \log \frac{1}{2} \geq \psi \geq A \log \frac{2}{3}, \quad \frac{A}{L} \geq-\psi^{\prime}(u) \geq \frac{A}{3 L}=2 C_{0}+1, \\
& \psi^{\prime \prime}(u) \geq \frac{2 \epsilon}{1-\epsilon}\left(\psi^{\prime}(u)\right)^{2}, \quad \text { for all } \epsilon \leq \frac{1}{2 A+1} .
\end{aligned}
$$

Suppose $\tilde{G}$ attains maximum a point $x_{0} \in X$ and a tangent direction $\xi_{0} \in T_{x_{0}} X$. In a standard way we construct normal coordinate system at $x_{0}$ and assume that $\xi_{0}=g_{1 \overline{1}}^{-1 / 2} \frac{\partial}{\partial z^{1}}$. We may also assume that $u_{i} \bar{j}$ is diagonal at $x_{0}$, i.e.,

$$
u_{i \bar{j}}\left(x_{0}\right)=\delta_{i j} u_{i \bar{i}}\left(x_{0}\right) \text {. }
$$

Then $\lambda_{i}:=1+u_{i \bar{i}}\left(x_{0}\right)$ are the eigenvalues of $\omega+d d^{c} u$ with respect to $\omega$ at $x_{0}$. Therefore, near $x_{0}$, the function

$$
G(z)=\log \left(1+g_{1 \overline{1}}^{-1} u_{1 \overline{1}}\right)+\varphi\left(|\nabla u|^{2}\right)+\psi(u)
$$

is well defined and has a maximum at $x_{0}$. At this moment we mention that $u_{1 \overline{1}}\left(x_{0}\right)$ is of the same size as $\Delta_{\omega} u\left(x_{0}\right)$ (meaning that for a numerical constant $C_{n}$ one has $C_{n}^{-1} u_{1 \overline{1}}\left(x_{0}\right) \leq$ $\left.\Delta_{\omega} u\left(x_{0}\right) \leq C_{n} u_{1 \overline{1}}\left(x_{0}\right)\right)$, since $\lambda_{i} \in \Gamma_{k}$ with $k \geq 2$ and hence $\sum_{j=2}^{n} \lambda_{i} \geq 0$. Note that in order to get the claimed global bound for the Laplacian in terms of the supremum of the gradient it is thus sufficient to bound $u_{1 \overline{1}}\left(x_{0}\right)$ by an expression which is of linear growth in $K$. To this end let us take the nonlinear operator

$$
S(\omega+i \partial \bar{\partial} u):=\log \sigma_{k}(\omega+i \partial \bar{\partial} u)
$$

which is different from $F=\sigma_{k}^{1 / k}$ used in [10]. Using the diagonality of $\omega$ and $u_{i} \bar{j}$ at $x_{0}$ we compute that

$$
S^{i \bar{j}}:=\frac{\partial S(\omega+i \partial \bar{\partial} u)}{\partial u_{i \bar{j}}}=\delta_{i j} \frac{\sigma_{k-1}(\lambda \mid i)}{\sigma_{k}(\lambda)} .
$$

At $x_{0}$ the second derivatives $S^{i \bar{j}, p \bar{q}}:=\frac{\partial^{2} S}{\partial u_{i} \bar{j} \partial u_{p \bar{q}}}$ are zero except in the following cases:

$$
S^{i \bar{i}, p \bar{p}}=\left(1-\delta_{i p}\right) \frac{\sigma_{k-2}(\lambda \mid i p)}{\sigma_{k}(\lambda)}-\frac{\sigma_{k-1}(\lambda \mid i) \sigma_{k-1}(\lambda \mid p)}{\sigma_{k}^{2}(\lambda)}
$$

and for $i \neq p$

$$
S^{i \bar{p}, p \bar{i}}=-\frac{\sigma_{k-2}(\lambda \mid i p)}{\sigma_{k}(\lambda)}
$$


Observe also that at $x_{0}$

$$
\sum_{i=1}^{n} S^{i \bar{i}}\left(1+u_{i \bar{i}}\right)=\sum_{i=1}^{n} S^{i \bar{i}} \lambda_{i}=k
$$

Differentiating the equation $S\left(\omega+d d^{c} u\right)=\log f$ and commuting the covariant derivatives we obtain the formulas (compare [10]) that at $x_{0}$

$$
\sum_{p=1}^{n} S^{p \bar{p}} u_{j p \bar{p}}=(\log f)_{j}+\sum_{p, q=1}^{n} u_{q} S^{p \bar{p}} R_{j \bar{q} p \bar{p}}
$$

and

$$
\sum_{p=1}^{n} S^{p \bar{p}} u_{1 \overline{1} p \bar{p}}=(\log f)_{1 \overline{1}}-\sum_{i, j, r, q=1}^{n} S^{i \bar{j}, r \bar{q}} u_{i \bar{j} 1} u_{r \bar{q} \overline{1}}+\sum_{p=1}^{n} S^{p \bar{p}}\left(u_{1 \overline{1}}-u_{p \bar{p}}\right) R_{1 \overline{1} p \bar{p}} .
$$

Returning to $G$ from the extremal property at $x_{0}$ we have the following formula

$$
0=G_{p}=\frac{u_{1 \overline{1} p}}{1+u_{1 \overline{1}}}+\varphi^{\prime} u_{p} u_{\bar{p} p}+\varphi^{\prime} \sum_{j=1}^{n} u_{j p} u_{\bar{p}}+\psi^{\prime} u_{p} .
$$

Also by diagonality, ellipticity, the equation itself and (3.14) we get

$$
\begin{aligned}
0 \geq & \sum_{p=1}^{n} S^{p \bar{p}} G_{p \bar{p}} \\
= & \sum_{p=1}^{n} \frac{S^{p \bar{p}} u_{1 \overline{1} p \bar{p}}}{1+u_{1 \overline{1}}}-\sum_{p=1}^{n} \frac{S^{p \bar{p}}\left|u_{1 \overline{1} p}\right|^{2}}{\left(1+u_{1 \overline{1}}\right)^{2}}+2 \varphi^{\prime} \operatorname{Re}\left[(\log f)_{\bar{j}} u_{j}\right]+\varphi^{\prime} \sum_{p, q, r=1}^{n} u_{\bar{r}} u_{q} S^{p \bar{p}} R_{p \bar{p}} \bar{q} \\
& +\sum_{p=1}^{n} \varphi^{\prime} S^{p \bar{p}}\left|u_{p \bar{p}}\right|^{2}+\sum_{p=1}^{n} \varphi^{\prime} S^{p \bar{p}} \sum_{j=1}^{n}\left|u_{j p}\right|^{2}+\varphi^{\prime \prime} \sum_{p=1}^{n} S^{p \bar{p}}\left|\sum_{j=1}^{n} u_{j p} u_{j}+u_{p} u_{p \bar{p}}\right|^{2} \\
& +\psi^{\prime \prime} \sum_{p=1}^{n} S^{p \bar{p}}\left|u_{p}\right|^{2}+\psi^{\prime} k-\psi^{\prime} \sum_{p=1}^{n} S^{p \bar{p}} .
\end{aligned}
$$

The first term can be estimated by exploiting (3.14), analogously to [10] we have

$$
\sum_{p=1}^{n} \frac{S^{p \bar{p}} u_{1 \overline{1} p \bar{p}}}{1+u_{1 \overline{1}}} \geq-\lambda_{1}^{-1} \sum_{i, j, r, q=1}^{n} S^{i \bar{j}, r \bar{q}} u_{i \bar{j} 1} u_{r \bar{q} \overline{1}}-C_{0} \sum_{p=1}^{n} S^{p \bar{p}}-C_{0} k+\frac{(\log f)_{1 \overline{1}}}{\lambda_{1}} .
$$

Denote $\mathcal{S}:=\sum_{p=1}^{n} S^{p \bar{p}}$. Then the fourth term in (3.16) can be estimated from below by

$$
\varphi^{\prime} \sum_{p, q, r=1}^{n} u_{\bar{r}} u_{q} S^{p \bar{p}} R_{p \bar{p} r \bar{q}} \geq-K \varphi^{\prime} \mathcal{S} C_{0} \geq-\frac{C_{0}}{2} \mathcal{S},
$$

where we used the property (3.7) of $\varphi^{\prime}$. The fifth term can be rewritten as

$$
\sum_{p=1}^{n} \varphi^{\prime} S^{p \bar{p}}\left|u_{p \bar{p}}\right|^{2}=\sum_{p=1}^{n} \varphi^{\prime} S^{p \bar{p}}\left|\lambda_{p}-1\right|^{2}=\sum_{p=1}^{n} \varphi^{\prime} S^{p \bar{p}} \lambda_{p}^{2}-2 \varphi^{\prime} k+\varphi^{\prime} \mathcal{S} .
$$


The sixth term is obviously nonnegative. So coupling (3.16) with (3.17), (3.18) and (3.19) we obtain

$$
\begin{aligned}
0 \geq & -\sum_{i, j, r, q=1}^{n} \frac{S^{i \bar{j}, r \bar{q}} u_{i \bar{j} 1} u_{r \bar{q} \overline{1}}}{1+u_{1 \overline{1}}}-\sum_{p=1}^{n} \frac{S^{p \bar{p}}\left|u_{1 \overline{1} p}\right|^{2}}{\left(1+u_{1 \overline{1}}\right)^{2}} \\
& +\psi^{\prime \prime} \sum_{p=1}^{n} S^{p \bar{p}}\left|u_{p}\right|^{2}+\varphi^{\prime \prime} \sum_{p=1}^{n} S^{p \bar{p}}\left|\sum_{j=1}^{n} u_{j p} u_{\bar{j}}+u_{p} u_{p \bar{p}}\right|^{2}+\varphi^{\prime} \sum_{p=1}^{n} S^{p \bar{p}} \lambda_{p}^{2} \\
& +\left(-\psi^{\prime}+\varphi^{\prime}-2 C_{0}\right) \mathcal{S}+\frac{(\log f)_{1 \overline{1}}}{\lambda_{1}}+2 \varphi^{\prime} \operatorname{Re}\left[(\log f)_{j} u_{j}\right]-\left(2 \varphi^{\prime}+\psi^{\prime}-C_{0}\right) k
\end{aligned}
$$

Up to now we have followed [10]. The big difference is that the last three terms, contained in the constant $C_{2}$ in [10], are not controllable from below in our setting. Define the constant $\delta:=\frac{1}{2 A+1}$. Let us divide the analysis into two separate cases:

Case 1 Suppose that $\lambda_{n}<-\delta \lambda_{1}$. Using the critical Eq. (3.15), we can exchange the term second term in (3.20) by

$$
-\sum_{p=1}^{n} \frac{S^{p \bar{p}}\left|u_{1 \overline{1}}\right|^{2}}{\left(1+u_{1 \overline{1}}\right)^{2}}=-\sum_{p=1}^{n} S^{p \bar{p}}\left|\varphi^{\prime} u_{p} u_{\bar{p} p}+\varphi^{\prime} \sum_{j=1}^{n} u_{j p} u_{\bar{p}}+\psi^{\prime} u_{p}\right|^{2}
$$

By Schwarz inequality this is further estimated from below by

$$
-\sum_{p=1}^{n} \frac{S^{p \bar{p}}\left|u_{1 \overline{1} p}\right|^{2}}{\left(1+u_{1 \overline{1}}\right)^{2}} \geq-2\left(\varphi^{\prime}\right)^{2} \sum_{p=1}^{n} S^{p \bar{p}}\left|\varphi^{\prime} u_{p} u_{\bar{p} p}+\varphi^{\prime} \sum_{j=1}^{n} u_{j p} u_{\bar{p}}\right|^{2}-2\left(\psi^{\prime}\right)^{2} \mathcal{S}|\nabla u|^{2} .
$$

Note that, by the choice of $\varphi$ (3.8), the first term above annihilates the fourth term in (3.20). The second one is bounded, using (3.9), by $-2\left(6 C_{0}+3\right)^{2} K \mathcal{S}$. Furthermore the first term in (3.20) is nonnegative by the concavity of the $S=\log \sigma_{k}$ operator, and the sixth term is also nonnegative by (3.7) and (3.9). Coupling the above inequalities we obtain

$$
\begin{aligned}
0 \geq & \varphi^{\prime} \sum_{p=1}^{n} S^{p \bar{p}} \lambda_{p}^{2}-18\left(2 C_{0}+1\right)^{2} K \mathcal{S}+\frac{(\log f)_{1 \overline{1}}}{\lambda_{1}}+2 \varphi^{\prime} \operatorname{Re}\left[(\log f)_{\bar{j}} u_{j}\right] \\
& -\left(2 \varphi^{\prime}+\psi^{\prime}-C_{0}\right) k .
\end{aligned}
$$

As $\varphi^{\prime} \geq \frac{1}{4 K}$, the first of these new terms is estimable by

$$
\varphi^{\prime} \sum_{p=1}^{n} S^{p \bar{p}} \lambda_{p}^{2} \geq \frac{1}{4 K} S^{n \bar{n}} \lambda_{n}^{2} \geq \frac{1}{4 n K} \mathcal{S} \lambda_{n}^{2} \geq \frac{1}{4 n K} \delta^{2} \mathcal{S} \lambda_{1}^{2}
$$

Here we used the case assumption and the fact that the coefficients $S^{j \bar{j}}$ increase in $j$. Next, using Corollary 2.5 and the fact that $\left\|f^{1 /(k-1)}\right\|_{\mathcal{C}^{1}},\left\|f^{1 /(k-1)}\right\|_{\mathcal{C}^{1,1}}$ are bounded, the last three terms in (3.21) can be estimated from below as

$$
\frac{(\log f)_{1 \overline{1}}}{\lambda_{1}}+2 \varphi^{\prime} \operatorname{Re}\left[(\log f)_{\bar{j}} u_{j}\right]-\left(2 \varphi^{\prime}+\psi^{\prime}-C_{0}\right) k \geq-\frac{C}{\lambda_{1} f^{1 /(k-1)}}-\frac{C}{\sqrt{K} f^{1 /(k-1)}}-C
$$


for some constant $C$ dependent on $C_{0}, k, B$ and $n$. Finally by MacLaurin inequality

$$
\begin{aligned}
\mathcal{S} & =\sum_{p=1}^{n} S^{p \bar{p}}=\sum_{p=1}^{n} \frac{\sigma_{k-1}(\lambda \mid p)}{\sigma_{k}(\lambda)}=(n-k+1) \frac{\sigma_{k-1}(\lambda)}{\sigma_{k}(\lambda)} \\
& \geq c(n, k) \frac{\sigma_{k}^{(k-2) /(k-1)} \sigma_{1}^{1 /(k-1)}}{\sigma_{k}} \geq c(n, k) \frac{\lambda_{1}^{1 /(k-1)}}{f^{1 /(k-1)}} .
\end{aligned}
$$

Therefore, multiplying both sides of the inequality (3.21) by $f^{1 /(k-1)}$, we get

$$
0 \geq c(n, k) \lambda_{1}^{1 /(k-1)}\left(\frac{\delta^{2}}{4 K n} \lambda_{1}^{2}-18\left(2 C_{0}+1\right)^{2} K\right)-C-C\left(1+\sup _{M} f^{1 /(k-1)}\right) .
$$

It follows that

$$
\lambda_{1}^{2} \leq C K^{2}+\frac{C K}{\delta^{2} \lambda_{1}^{1 /(k-1)}}
$$

for some $C$ under control.

Case 2 Assume $\lambda_{n} \geq-\delta \lambda_{1}$. Exactly as in [10], we consider

$$
I:=\left\{i \in\{1, \ldots, n\} \mid \sigma_{k-1}(\lambda \mid i)>\delta^{-1} \sigma_{k-1}(\lambda \mid 1)\right\}
$$

As $\delta^{-1}=2 A+1 \geq 7, i=1$ does not belong to $I$. Returning to our setting we get that $p \in I$ if and only if

$$
S^{p \bar{p}}>\delta^{-1} S^{1 \overline{1}}
$$

Then exploiting (3.15) and the Schwarz inequality we have

$$
\begin{aligned}
& -\sum_{p \in\{1, \ldots, n\} \backslash I} \frac{S^{p \bar{p}}\left|u_{1 \overline{1} p}\right|^{2}}{\left(1+u_{1 \overline{1}}\right)^{2}} \\
& \geq-2\left(\varphi^{\prime}\right)^{2} \sum_{p \in\{1, \ldots, n\} \backslash I} S^{p \bar{p}}\left|u_{p} u_{p \bar{p}}+\sum_{j=1}^{n} u_{j p} u_{\bar{p}}\right|^{2}-2\left(\psi^{\prime}\right)^{2} \sum_{p \in\{1, \ldots, n\} \backslash I} S^{p \bar{p}}\left|u_{p}\right|^{2} \\
& \geq-2\left(\varphi^{\prime}\right)^{2} \sum_{p \in\{1, \ldots, n\} \backslash I} S^{p \bar{p}}\left|u_{p} u_{p \bar{p}}+\sum_{j=1}^{n} u_{j p} u_{\bar{p}}\right|^{2}-18\left(2 C_{0}+1\right)^{2} K S^{1 \overline{1}} .
\end{aligned}
$$

Using the same strategy as in case 1, the first term annihilates the following term in (3.20):

$$
\varphi^{\prime \prime} \sum_{p \in\{1, \ldots, n\} \backslash I} S^{p \bar{p}}\left|u_{p} u_{p \bar{p}}+\sum_{j=1}^{n} u_{j p} u_{\bar{p}}\right|^{2}
$$


What remains from (3.20) can be written as

$$
\begin{aligned}
0 \geq & -\sum_{i, j, r, q=1}^{n} \frac{S^{i \bar{j}, r \bar{q}} u_{i \bar{j} 1} u_{r \bar{q} \overline{1}}}{1+u_{1 \overline{1}}}-\sum_{p \in I} \frac{S^{p \bar{p}}\left|u_{1 \overline{1} p}\right|^{2}}{\left(1+u_{1 \overline{1}}\right)^{2}}+\left(-\psi^{\prime}+\varphi^{\prime}-2 C_{0}\right) \mathcal{S} \\
& +\varphi^{\prime \prime} \sum_{p \in I} S^{p \bar{p}}\left|u_{p} u_{p \bar{p}}+\sum_{j=1}^{n} u_{j p} u_{\bar{p}}\right|^{2}+\psi^{\prime \prime} \sum_{p=1}^{n} S^{p \bar{p}}\left|u_{p}\right|^{2}+\frac{1}{4 K} \sum_{p=1}^{n} S^{p \bar{p}} \lambda_{p}^{2} \\
& +\frac{(\log f)_{1 \overline{1}}}{\lambda_{1}}+2 \varphi^{\prime} \operatorname{Re}\left[(\log f)_{j} u_{j}\right]-\left(2 \varphi^{\prime}+\psi^{\prime}-C_{0}\right) k-18\left(2 C_{0}+1\right)^{2} K S^{1 \overline{1}} .
\end{aligned}
$$

If $\lambda_{1}^{2} \geq\left[12\left(2 C_{0}+1\right) K\right]^{2}$ (which we can safely assume a priori for otherwise we are through) the last term can be absorbed by the sixth one. Also $-\psi^{\prime}+\varphi^{\prime}-2 C_{0} \geq 1$, therefore the previous estimate is reduced to

$$
\begin{aligned}
0 \geq & -\sum_{i, j, r, q=1}^{n} \frac{S^{i \bar{j}, r \bar{q}} u_{i \bar{j} 1} u_{r \bar{q} \overline{1}}}{1+u_{1 \overline{1}}}-\sum_{p \in I} \frac{S^{p \bar{p}}\left|u_{1 \overline{1}}\right|^{2}}{\left(1+u_{1 \overline{1}}\right)^{2}}+\varphi^{\prime \prime} \sum_{p \in I} S^{p \bar{p}}\left|u_{p} u_{p \bar{p}}+\sum_{j=1}^{n} u_{j p} u_{\bar{p}}\right|^{2} \\
& +\psi^{\prime \prime} \sum_{p=1}^{n} S^{p \bar{p}}\left|u_{p}\right|^{2}+\frac{1}{8 K} \sum_{p=1}^{n} S^{p \bar{p}} \lambda_{p}^{2}+\mathcal{S}+\frac{(\log f)_{1 \overline{1}}}{\lambda_{1}}+2 \varphi^{\prime} \operatorname{Re}\left[(\log f)_{\bar{j}} u_{j}\right] \\
& -\left(2 \varphi^{\prime}+\psi^{\prime}-C_{0}\right) k .
\end{aligned}
$$

As as case 1, the last three terms can be estimated by $-\frac{C}{f^{1 /(k-1)}}$ for some constant $C$ dependent on $B, n, C_{0}$ and $k$. So if the first four terms add up to something nonnegative then we end up with

$$
\frac{C_{3}}{f^{1 /(k-1)}} \geq \mathcal{S}+\frac{1}{8 K} \sum_{p=1}^{n} S^{p \bar{p}} \lambda_{p}^{2} .
$$

This together with (3.22) imply $\lambda_{1} \leq C$.

What remains is to prove the non-negativity of

$$
\begin{aligned}
& -\sum_{i, j, r, q=1}^{n} \frac{S^{i \bar{j}, r \bar{q}} u_{i \bar{j} 1} u_{r \bar{q} \overline{1}}}{1+u_{1 \overline{1}}}-\sum_{p \in I} \frac{S^{p \bar{p}}\left|u_{1 \overline{1} p}\right|^{2}}{\left(1+u_{1 \overline{1}}\right)^{2}}+\varphi^{\prime \prime} \sum_{p \in I} S^{p \bar{p}}\left|u_{p} u_{p \bar{p}}+\sum_{j=1}^{n} u_{j p} u_{\bar{p}}\right|^{2} \\
& +\psi^{\prime \prime} \sum_{p=1}^{n} S^{p \bar{p}}\left|u_{p}\right|^{2} .
\end{aligned}
$$

Exploiting (3.15) and Proposition 2.3 from [10] the last two terms can be estimated from below by

$$
\sum_{p \in I} S^{p \bar{p}}\left[2\left(\varphi^{\prime}\right)^{2}\left|u_{p} u_{p \bar{p}}+\sum_{j=1}^{n} u_{j p} u_{\bar{p}}\right|^{2}+\frac{2 \delta}{1-\delta}\left|u_{p}\right|^{2}\right] \geq 2 \delta \sum_{p \in I} \frac{S^{p \bar{p}}\left|u_{1 \overline{1} p}\right|^{2}}{\left(1+u_{1 \overline{1}}\right)^{2}} .
$$

On the other hand, the concavity of the $S=\log \sigma_{k}$ operator yields that the first term is controlled from below by

$$
-\sum_{i, j, r, q=1}^{n} \frac{S^{i \bar{j}, r \bar{q}} u_{i \bar{j} 1} u_{r \bar{q} \overline{1}}}{1+u_{1 \overline{1}}} \geq-\sum_{p \in I} \frac{S^{p \overline{1}, 1 \bar{p}}\left|u_{1 \overline{1} p}\right|^{2}}{1+u_{1 \overline{1}}} .
$$


Therefore, the inequality to-be-proven will be satisfied if

$$
-S^{p \overline{1}, 1 \bar{p}} \geq(1-2 \delta) \frac{S^{p \bar{p}}}{\lambda_{1}},
$$

for each $p \in I$. Exploiting the formulas for $S^{p \overline{1}, 1 \bar{p}}$ and $S^{p \bar{p}}$ this is in turn equivalent to

$$
\frac{\sigma_{k-2}(\lambda \mid 1 p)}{\sigma_{k}} \geq(1-2 \delta) \frac{\sigma_{k-1}(\lambda \mid p)}{\lambda_{1} \sigma_{k}} .
$$

But the inequality above is exactly the inequality proven in [10] (page 559). Indeed, the inequality can be rewritten as

$$
\left(2 \delta \lambda_{1}+(1-2 \delta) \lambda_{p}\right) \sigma_{k-1}(\lambda \mid p) \geq \lambda_{1} \sigma_{k-1}(\lambda \mid 1)
$$

and the latter one holds due to the case assumptions $\lambda_{p} \geq \lambda_{n}>-\delta \lambda_{1}$ and $\sigma_{k-1}(\lambda \mid p) \geq$ $\delta^{-1} \sigma_{k-1}(\lambda \mid p)$. Thus the claimed inequality is proven.

\section{Examples}

In this section we shall investigate the examples from Proposition 1.3 in the real and complex domains. We will also deal with the complex compact manifold case.

As mentioned in the Introduction, it was stated in [11] that a modification of the argument from the Monge-Ampère case (see [23]) shows that the exponent $1 /(k-1)$ on the right hand side cannot be improved any further. An important feature of these examples is that they are separately radial in all but one of the coordinates and radial in the distinguished coordinate. In the convex setting this means that

$$
u\left(x^{\prime}, x_{n}\right)=u\left(y^{\prime}, y_{n}\right), \text { whenever }\left|x^{\prime}\right|=\left|y^{\prime}\right| \text { and }\left|x_{n}\right|=\left|y_{n}\right| .
$$

Here, we use the notation $x=\left(x^{\prime}, x_{n}\right)=\left(x_{1}, \ldots, x_{n}\right)$.

Note that, for convex $u$, this implies that $u$ is increasing in the both radial directions. The same observation holds for a pluri-subharmonic function $v\left(z^{\prime}, z_{n}\right)$ radial in both directions and this was heavily used in [19]. What makes the $k$-Hessian case different is that a priori such a $k$-convex function will be increasing in the directions $x^{\prime}$ only as the 2-convex example

$$
u\left(x^{\prime}, x_{3}\right)=u\left(x_{1}, x_{2}, x_{3}\right)=3\left(x_{1}^{2}+x_{2}^{2}\right)-x_{3}^{2}
$$

shows. We will nevertheless prove an additional lemma showing that our examples are indeed increasing in the radial $x_{n}$ (respectively $z_{n}$ ) directions. Given this lemma, the proof is indeed analogous to the one in [23] in the $k$-convex case and to [19] in the $k$-subharmonic case. Our lemma can also be generalized to work on $\mathbb{P}^{n-1} \times \mathbb{P}^{1}$ equipped with the Fubini-Study product metric and thus provides examples in the case of compact Kähler manifolds. Below we provide the full details.

\subsection{Examples in the real setting}

In this subsection we fix $1<k \leq n$. We will work in the unit ball $B=B(0,1)$ in $\mathbb{R}^{n}$. The following lemma is crucial for our construction. 
Lemma 4.1 Let $u$ be a continuous $k$-convex function on $B$ which is constant on $\partial B$ and it depends only on $\left|x_{n}\right|$ and $\left|x^{\prime}\right|$. Assume that $F=S_{k}\left(D^{2} u\right)$ is (weakly) decreasing with respect to $x_{n}$. Then $u$ is weakly increasing with respect to $\left|x_{n}\right|$. In particular,

$$
\inf _{B} u=u(0) .
$$

Proof Observe that for $k=n$ this follows simply from the convexity of $u$, but for $k<n$ we have to work harder.

Note that $u$ is radially invariant in the $x_{n}$ direction, it suffices to prove that for each $x^{\prime} \in \mathbb{R}^{n-1},\left|x^{\prime}\right|<1$ the function $t \rightarrow u\left(x^{\prime}, t\right)$ is increasing on the interval $\left(0, \sqrt{1-\left|x^{\prime}\right|^{2}}\right)$. For any $\varepsilon>0, \delta \geq 0$, we define

$$
v_{\varepsilon}\left(x^{\prime}, x_{n}\right)=u\left(x^{\prime}, x_{n}\right)+\varepsilon|x|^{2}
$$

and

$$
w_{\varepsilon, \delta}\left(x^{\prime}, x_{n}\right)=u\left(x^{\prime}, x_{n}+\delta\right)+2 \varepsilon .
$$

Then, our goal is to show that $v_{\varepsilon}\left(x^{\prime}, x_{n}\right) \leq w_{\varepsilon, \delta}\left(x^{\prime}, x_{n}\right)$ for any $\left(x^{\prime}, x_{n}\right) \in B$ such that $\left(x^{\prime}, x_{n}+\delta\right) \in B$ and $x_{n}>0$. If this holds, taking $\varepsilon \rightarrow 0$, we obtain

$$
u\left(x^{\prime}, x_{n}\right) \leq u\left(x^{\prime}, x_{n}+\delta\right)
$$

for any $\delta>0$ and $0<x_{n}<x_{n}+\delta<\sqrt{1-\left|x^{\prime}\right|^{2}}$.

To obtain the desired inequality, we first observe that, using the assumption that $S_{k}\left(D^{2} u\right)$ is (weakly) decreasing with respect to $\left|x_{n}\right|$, we have

$$
S_{k}\left(D^{2} v_{\varepsilon}\right)=S_{k}\left(D^{2} u\left(x^{\prime}, x_{n}\right)+2 \varepsilon I_{n}\right)>S_{k}\left(D^{2} u\left(x^{\prime}, x_{n}\right)\right) \geq S_{k}\left(D^{2} w_{\varepsilon, \delta}\right)
$$

on $S_{\delta}=\left\{\left(x^{\prime}, x_{n}\right): x_{n}>-\delta / 2,\left(x^{\prime}, x_{n}+\delta\right) \in B\right\} \subset B$.

For $\left(x^{\prime}, x_{n}\right) \in \partial S_{\delta} \cap\left\{x_{n}>-\frac{\delta}{2}\right\}$ we know that $\left(x^{\prime}, x_{n}+\delta\right) \in \partial B$. Therefore,

$$
w_{\varepsilon, \delta}\left(x^{\prime}, x_{n}\right)=u\left(x^{\prime}, x_{n}+\delta\right)+2 \varepsilon=\max _{B} u+2 \varepsilon .
$$

Here we used that fact that $u$ is $k$-convex and equals to constant on $\partial B$ and hence $u$ attains its maximum on $\partial B$. Recalling the definition of $v_{\varepsilon}$, we have

$$
w_{\varepsilon, \delta}(x)>v_{\varepsilon}(x), \text { for } x \in \partial S_{\delta} \cap\left\{x_{n} \geq 0\right\} .
$$

On the other hand, for any point $q=\left(q^{\prime}, q_{n}\right) \in B \cap\left\{x_{n}=-\frac{\delta}{2}\right\}=\partial S_{\delta} \backslash\left(\partial S_{\delta} \cap\left\{x_{n}>-\frac{\delta}{2}\right\}\right)$, we compute

$$
w_{\varepsilon, \delta}(q)=w_{\varepsilon, 0}\left(q^{\prime},-q_{n}\right)>v_{\varepsilon}\left(q^{\prime},-q_{n}\right)=v_{\varepsilon}(q) .
$$

Therefore, we have

$$
w_{\varepsilon, \delta}(x)>v_{\varepsilon}(x) \text {, on } \partial S_{\delta} .
$$

By (4.2) and the comparison principle (Theorem 2.1) we obtain

$$
w_{\varepsilon, \delta}>v_{\varepsilon} \text { on } S_{\delta} .
$$

Letting $\varepsilon \rightarrow 0$, we get that $u$ is weakly increasing in $\left|x_{n}\right|$. Finally (4.1) follows from the sub-harmonicity of $u$ with respect to $x^{\prime}$. 
Given Lemma 4.1, the construction of the example and its justification follow closely the argument in [23]. We provide the details for the sake of completeness. Let

$$
\eta(t)= \begin{cases}\exp \left(-1 /\left(1-t^{2}\right)\right) & t<1 \\ 0 & t \geq 1\end{cases}
$$

For $a, b \in \mathbb{R}, a>1$ define

$$
F(x)= \begin{cases}\eta\left(\frac{\left|x_{n}\right|}{\left|x^{\prime}\right|^{a}}\right)\left|x^{\prime}\right|^{b} & \text { if } x^{\prime} \neq 0 \\ 0 & \text { if } x^{\prime}=0\end{cases}
$$

Example 4.2 If $0<b<2(k-1)(a-1)$, then the k-convex solution $u$ of the Dirichlet problem

$$
\begin{cases}S_{k}\left(D^{2} u\right)=F(x) & \text { in } B \\ u=0 & \text { on } \partial B\end{cases}
$$

is not $\mathcal{C}^{1,1}$ in any neighbourhood of 0 . Furthermore if $b=2(k-1)(a-2)$, then $F^{\gamma} \in \mathcal{C}^{1,1}(B)$ for $\gamma>\frac{1}{k-1}+\frac{2}{(k-1)(a-2)}$. In particular, taking $a \rightarrow \infty$ we obtain that no exponent larger than $1 /(k-1)$ could yield $\mathcal{C}^{1,1}$ solutions in general.

Proof The comparison principle implies that the solution is unique. Because of the rotational invariance of the data the solution has to depend only on $\left|x^{\prime}\right|$ and $\left|x_{n}\right|$, i.e. it has to be radial both in the $x^{\prime}$ and the $x_{n}$ direction. By Lemma 4.1 it is increasing separately in $\left|x^{\prime}\right|$ and in $\left|x_{n}\right|$.

Let $\varepsilon>0$ be such that $\varepsilon^{2}+\varepsilon^{2 / a}<1$. Define the domain

$$
P=\left\{\left(x^{\prime}, x_{n}\right):\left|x^{\prime}\right|<\varepsilon^{1 / a},\left|x_{n}\right|<\varepsilon\right\}
$$

and the function

$$
v(x)=\left(\frac{x_{1}-\frac{1}{2} \varepsilon^{1 / a}}{\frac{1}{4} \varepsilon^{1 / a}}\right)^{2}+\sum_{k=2}^{n-1}\left(\frac{x_{k}}{\frac{1}{2} \varepsilon^{1 / a}}\right)^{2}+\left(\frac{x_{n}}{\frac{1}{4^{a+1}} \varepsilon}\right)^{2}-1 .
$$

By computation we have

$$
E:=\{x \in B: v<0\} \subset P .
$$

On the other hand

$$
\inf _{E} F \geq \eta(1 / 4) 4^{-b} \varepsilon^{b / a}
$$

Observe also that for some positive constant $c_{1}$ (independent on $\varepsilon$ ) the following inequality holds

$$
S_{k}\left(D^{2} v\right) \leq c_{1} \varepsilon^{-2-2(k-1) / a} .
$$

Then it is possible to choose another constant $c_{2}$ (also independent on $\varepsilon$ ) such that

$$
S_{k}\left(D^{2}\left(c_{2} \varepsilon^{\frac{2 a+2(k-1)+b}{k a}} v+\sup _{P} u\right)\right) \leq \inf _{E} F .
$$

By the comparison principle

$$
c_{2} \varepsilon^{\frac{2 a+2(k-1)+b}{k a}} v+\sup _{P} u \geq u, \text { on } P
$$


and we obtain

$$
\begin{aligned}
u(0) & \leq u\left(\frac{1}{2} \varepsilon^{1 / a}, 0, \ldots, 0\right) \leq \sup _{P} u-c_{2} \varepsilon^{\frac{2 a+2(k-1)+b}{k a}} \\
& =u\left(\varepsilon^{1 / a}, 0, \ldots, 0, \varepsilon\right)-c_{2} \varepsilon^{\frac{2 a+2(k-1)+b}{k a}} .
\end{aligned}
$$

For the last equality, we used the fact that $u$ obtains its maximum on $\partial P$ since $u$ is radial and increasing in both $x^{\prime}$ and $x_{n}$ directions. Denote

$$
s(t)=u\left(0^{\prime}, t\right), \quad \text { and } \ell(t)=u\left(t^{1 / a}, 0, \ldots, 0, t\right)
$$

for $t \in[0,1]$. We clearly have $s \leq \ell$ since $u$ is increasing in the $\left|x^{\prime}\right|$ direction.

Assume that $s<\ell$ on some interval $(c, d)$. Then, for any $\varepsilon_{1}, \varepsilon_{2} \in(c, d)$ with $\varepsilon_{2}-\varepsilon_{1}>0$ small enough, we can find an affine function $w$ dependent only on $x_{n}$, such that $w\left(x^{\prime}, t\right)<\ell(t)$ for any $t \in\left(\varepsilon_{1}, \varepsilon_{2}\right)$ and

$$
w\left(0^{\prime}, \varepsilon_{1}\right)=u\left(0^{\prime}, \varepsilon_{1}\right)=s\left(\varepsilon_{1}\right), w\left(0^{\prime}, \varepsilon_{2}\right)=u\left(0^{\prime}, \varepsilon_{2}\right)=s\left(\varepsilon_{2}\right) .
$$

Then, by the monotonicity of $u$ in the $\left|x^{\prime}\right|$ direction again, we have

$$
w\left(x^{\prime}, x_{n}\right) \leq u\left(x^{\prime}, x_{n}\right) \text { on } \partial\left(\{F=0\} \cap\left\{x_{n} \in\left(\varepsilon_{1}, \varepsilon_{2}\right)\right\}\right) .
$$

On the other hand, by the definition of $F$, we have $S_{k}\left(D^{2} u\right)=0=S_{k}\left(D^{2} w\right)$ on $\{F=$ $0\} \cap\left\{x_{n} \in\left(\varepsilon_{1}, \varepsilon_{2}\right)\right\}$. Then, the comparison principle implies

$$
w\left(x^{\prime}, x_{n}\right) \leq u\left(x^{\prime}, x_{n}\right) \operatorname{in}\{F=0\} \cap\left\{x_{n} \in\left(\varepsilon_{1}, \varepsilon_{2}\right)\right\}
$$

for any small interval $\left(\varepsilon_{1}, \varepsilon_{2}\right) \in(c, d)$. In particular, $w\left(0^{\prime}, t\right) \leq u\left(0^{\prime}, t\right)=s(t)$ for any $t \in\left(\varepsilon_{1}, \varepsilon_{2}\right)$. Thus $s(t)$ is weakly concave on $(c, d)$.

Now assume that $u$ is $\mathcal{C}^{1,1}$ in a neighbourhood of 0 . Then $s^{\prime}(0)=0$. We claim that $s$ cannot be concave in any interval of the type $(0, r)$. Indeed, if $s(t)$ is weakly concave on some interval $(0, r)$, then it follows that $s^{\prime}(t) \leq 0$ for $t \in(0, r)$. On the other hand, by Lemma 4.1, we have $s^{\prime}(t) \geq 0$. Therefore, $s^{\prime}(t) \equiv 0$ and hence $s(t)$ is constant on $(0, r)$. Taking largest such $r$ (which is strictly less than one for otherwise the function would be globally constant), we have $s(r)<\ell(r)$ as $u$ is not constant in a neighbourhood of zero. But then applying the above argument around the point $r$, we would obtain that $s$ is concave at $r$. This contradicts with the fact that $s$ is constant to the left of $r$ and strictly increases to the right of $r$. This proves the claim.

Then, it follows that the strict inequality $s(t)<\ell(t)$ can not hold in any interval of the type $(0, r)$. Thus there is a sequence $\varepsilon_{m}$ decreasing to 0 such that

$$
u\left(0, \varepsilon_{m}\right)=s\left(\varepsilon_{m}\right)=l\left(\varepsilon_{m}\right) \geq u(0)+c_{2} \varepsilon_{m}^{\frac{2 a+2(k-1)+b}{k a}}
$$

and we can conclude

$$
u\left(0, \varepsilon_{m}\right)-u(0) \geq c_{2} \varepsilon_{m}^{2-\theta / a k},
$$

where $\theta=2(k-1)(a-1)-b$. This contradicts the assumption that $u \in \mathcal{C}^{1,1}$ around 0 .

\subsection{Compact Kähler manifold case}

Now we deal with the compact Kähler manifold case. The construction is similar to the real case and the main technical difficulty is that we have to replace the translation operators 
with suitable automorphisms of the Kähler manifold. These automorphisms will furthermore preserve the Kähler form.

We fix $1<k \leq n$ in what follows. The examples will be constructed on $\mathbb{P}^{n-1} \times \mathbb{P}^{1}$ equipped with the product metric $\omega=\omega_{F S}^{\prime}+\omega_{F S}$ with $\omega_{F S}$ denoting the Fubini-Study metrics on each factor. For $z \in \mathbb{C}^{n}$ we split the coordinates and write $z=\left(z^{\prime}, z_{n}\right) \in \mathbb{C}^{n-1} \times \mathbb{C}$ which we identify in the usual way as a subset (the affine chart) of $\mathbb{P}^{n-1} \times \mathbb{P}^{1}$. On this affine chart we have $\omega_{F S}^{\prime}=i \partial \bar{\partial}\left(\frac{1}{2} \log \left(1+\left|z^{\prime}\right|^{2}\right)\right)$ and $\omega_{F S}=i \partial \bar{\partial}\left(\frac{1}{2} \log \left(1+\left|z_{n}\right|^{2}\right)\right)$. The following complex analogue of Lemma 4.1 is crucial for the construction.

Lemma 4.3 Let $\varphi \in \mathcal{S H}_{k}\left(\mathbb{P}^{n-1} \times \mathbb{P}^{1}, \omega\right)$ be a continuous function such that

$$
(\omega+i \partial \bar{\partial} \varphi)^{k} \wedge \omega^{n-k}=f \omega^{n} .
$$

Moreover, assume that

1. for any $r>0$ the set $\left\{\left(z^{\prime}, z_{n}\right) \in \mathbb{C}^{n-1} \times \mathbb{C}:\left|z_{n}\right| \leq r, f\left(z^{\prime}, z_{n}\right)=0\right\}$ is bounded;

2. $\varphi \mid \mathbb{C}^{n}$ (and hence $f$ ) depends only on $\left|z^{\prime}\right|$ and $\left|z_{n}\right|$ on the affine chart;

3. $f\left(z^{\prime}, z_{n}\right)$ is strictly decreasing in $\left|z_{n}\right|$ for all fixed $z^{\prime}$ such that $f\left(z^{\prime}, z_{n}\right)>0$.

Then the function $\varphi$ is increasing with respect to $\left|z_{n}\right|$.

Proof Denote by $t_{\alpha}$ and $T_{\alpha}$ the automorphisms of $\mathbb{P}^{1}$ and $\mathbb{P}^{n-1} \times \mathbb{P}^{1}$ respectively given by

$$
\begin{gathered}
t_{\alpha}\left(\left[w_{0}: w_{1}\right]\right)=\left[\cos (\alpha) w_{0}-\sin (\alpha) w_{1}: \sin (\alpha) w_{0}+\cos (\alpha) w_{1}\right] ; \\
T_{\alpha}\left(\left[z_{0}: \cdots z_{n-1}\right] \times\left[w_{0}: w_{1}\right]\right)=\left[z_{0}: \cdots: z_{n-1}\right] \times t_{\alpha}\left(\left[w_{0}: w_{1}\right]\right) .
\end{gathered}
$$

We would like to point out that $t_{\alpha}$ preserves $\omega_{F S}$ while $T_{\alpha}$ preserves the product metric $\omega$. Moreover, on the affine chart of $\mathbb{P}^{1}, t_{\alpha}$ reads

$$
t_{\alpha}(z)=\frac{z+\tan \alpha}{1-z \tan \alpha} .
$$

Choose now $\varepsilon>0$ and fix an angle $\alpha \in\left(0, \frac{\pi}{4}\right]$. Let $W=\{z \in \mathbb{C}: \operatorname{Re} z \geq 0\} \cup\{\infty\} \subset \mathbb{P}^{1}$ and $E=T_{\frac{\alpha}{2}}^{-1}\left(\mathbb{P}^{n-1} \times W\right)$. For $\left(z^{\prime}, z_{n}\right) \in \operatorname{int}(E)$ we have

$$
t_{\alpha}\left(z_{n}\right)=\infty \text { or }\left|z_{n}\right|<\left|t_{\alpha}\left(z_{n}\right)\right| \text {. }
$$

Let $\psi: \mathbb{P}^{n-1} \times \mathbb{P}^{1} \rightarrow \mathbb{R}$ be a continuous function given by

$$
\psi\left(z^{\prime}, z_{n}\right)=\left(\varphi \circ T_{\alpha}\right)\left(z^{\prime}, z_{n}\right)+\varepsilon .
$$

For $z \in \partial E$, we have $\left|z_{n}\right|=\left|t_{\alpha}\left(z_{n}\right)\right|$ and hence $\varphi(z)=\varphi\left(T_{\alpha}(z)\right)<\psi(z)$. Thus, for any $\delta>0$ small enough, the set

$$
D:=\{\varphi-\delta>\psi\} \cap E
$$

is relatively compact in $\operatorname{int}(E)$. The monotonicity properties of $f$ imply that

$$
f(z) \geq f\left(T_{\alpha}(z)\right) \text { for } z \in \bar{E} .
$$

The comparison principle (2.3) results in

$\int_{D} f \omega^{n} \geq \int_{D} f \circ T_{\alpha} \omega^{n}=\int_{D}(\omega+i \partial \bar{\partial} \psi)^{k} \wedge \omega^{n-k} \geq \int_{D}(\omega+i \partial \bar{\partial} \varphi)^{k} \wedge \omega^{n-k}=\int_{D} f \omega^{n}$.

Together with assumption (3) and (4.6), this gives us $f=0$ on $D$. We wish to point out that, contrary to the local setting, we cannot deduct the emptiness of $D$ at this stage since 
we do not know whether $D$ is contained in some affine chart. To this end we use assumption (1). Note that the projection of $E$ onto the $\mathbb{P}^{1}$ factor is a bounded subset of the affine chart. By assumption (1) we get that $D$ is bounded. Then the comparison principle for bounded domains implies that $D$ is empty.

Let $\eta$ be as in the real case. For $a \geq 1, b \in \mathbb{R}$ and $z \in \mathbb{C}^{n}$, define

$$
f(z)=A \exp \left(-|z|^{2}\right) \eta\left(\frac{\left|z_{n}\right|}{\left|z^{\prime}\right|^{a}}\right)\left|z^{\prime}\right|^{b}
$$

and extend $f$ by zero on the divisors of infinity so that $f$ is a function on $\mathbb{P}^{n-1} \times \mathbb{P}^{1}$. Here, $\eta(t)$ is given in (4.3) and the constant $A>0$ is chosen such that

$$
\int_{\mathbb{P}^{n-1} \times \mathbb{P}^{1}} f \omega^{n}=\int_{\mathbb{P}^{n-1} \times \mathbb{P}^{1}} \omega^{n} .
$$

Lemma 4.4 If $\varphi \in \mathcal{S H}_{k}\left(\mathbb{P}^{n-1} \times \mathbb{P}^{1}, \omega\right)$ is the unique continuous solution to the equation

$$
(\omega+i \partial \bar{\partial} \varphi)^{k} \wedge \omega^{n-k}=f \omega^{n}
$$

on $\mathbb{P}^{n-1} \times \mathbb{P}^{1}$, satisfying $\varphi(0)=0$. Then there exist a constant $c>0$ and a sequence $\varepsilon_{m}>0$ which decreases to 0 , such that

$$
u\left(0, \varepsilon_{m}\right) \geq c \varepsilon_{m}^{\theta},
$$

where $\theta=\frac{2 a+2 k-2+b}{k a}$ and $u=\left.\varphi\right|_{\mathbb{C}^{n}}+\frac{1}{2} \log \left(1+\left|z^{\prime}\right|^{2}\right)+\frac{1}{2} \log \left(1+\left|z_{n}\right|^{2}\right)$.

Proof We remark that the solution is unique (uniqueness for normalized solutions to complex Hessian equations holds in much greater generality that we need here, see for example [4]). Just as in the real case this implies that it depends only on $\left|z^{\prime}\right|$ and $\left|z_{n}\right|$ and thus by definition $u$ depends only on $\left|z^{\prime}\right|$ and $\left|z_{n}\right|$ too. By sub-harmonicity $u$ is increasing with respect to $\left|z^{\prime}\right|$ and by Lemma 4.3 it is strictly increasing in $\left|z_{n}\right|$ as the function $f$ satisfies all the assumptions in that lemma.

From now on, we restrict our attention to the affine chart. Let $\varepsilon>0$ be such that $\varepsilon^{2}+\varepsilon^{2 / a} \leq$ 1. Let

$$
P=\left\{\left(z^{\prime}, z_{n}\right):\left|z^{\prime}\right|<\varepsilon^{1 / a},\left|z_{n}\right|<\varepsilon\right\}
$$

and

$$
v(x)=\left(\frac{\operatorname{Re} z_{1}-\frac{1}{2} \varepsilon^{1 / a}}{\frac{1}{4} \varepsilon^{1 / a}}\right)^{2}+\left(\frac{\operatorname{Im} z_{1}}{\frac{1}{4} \varepsilon^{1 / a}}\right)^{2}+\sum_{k=2}^{n-1}\left(\frac{\left|z_{k}\right|}{\frac{1}{4} \varepsilon^{1 / a}}\right)^{2}+\left(\frac{\left|z_{n}\right|}{\frac{1}{4^{a+1}} \varepsilon}\right)^{2} .
$$

Then

$$
E=\{x \in B: v<1\} \subset P .
$$

We have

$$
\inf _{E} f \geq \exp (-2) \eta(1 / 4) 4^{-b} \varepsilon^{b / a}
$$

and we can choose an absolute constant $c_{1}$ such that

$$
(i \partial \bar{\partial} v)^{k} \wedge \omega^{n-k} \leq c_{1} \varepsilon^{-2-2(k-1) / a} \omega^{n} \text { on } P \text {. }
$$


Then it is possible to choose a constant $c_{2}$ independent on $\varepsilon$ such that

$$
\left(i \partial \bar{\partial}\left(\varepsilon^{\frac{2 a+2(k-1)+b}{k a}} v\right)\right)^{k} \wedge \omega^{n-k} \leq c_{2} f \omega^{n} \text { on } E .
$$

By the comparison principle we obtain

$$
u\left(\varepsilon^{1 / a}, 0, \ldots, 0, \varepsilon\right)=\sup _{P} u \geq \sup _{E} u \geq \inf _{\partial E} c_{2} \varepsilon^{\frac{2 a+2(k-1)+b}{k a}} v=c_{2} \varepsilon^{\frac{2 a+2(k-1)+b}{k a}} .
$$

For $t \in \mathbb{R}$, we define

$$
s(t)=u\left(0, e^{t}\right), \quad \text { and } \quad \ell(t)=u\left(e^{t / a}, 0, \ldots, 0, e^{t}\right) .
$$

We have $s \leq \ell$. Follow the same argument as in the real case, we can obtain that if $s<\ell$ on some interval $(c, d)$ then $s$ is weakly concave on $(c, d)$. However, we also know that $s$ is strictly increasing and

$$
\lim _{t \rightarrow-\infty} s(t)=0
$$

and this imply $s$ can not be weakly concave in any interval $(-\infty, r)$. Therefore, there is a sequence $t_{m} \searrow-\infty$ such that $s\left(t_{m}\right)=\ell\left(t_{m}\right)$. Taking $\varepsilon_{m}=e^{t_{m}}$ and using (4.11) we obtain the Lemma.

Observe that in the argument above the parameter $b$ can be negative and then the right hand side is merely $L^{p}$ integrable. In such a case a result from [5] shows that local solutions are bounded for $L^{p}$ integrable right hand side provided $p>\frac{n}{k}$. It is natural to ask the following question:

Question 4.5 Consider the $k$-Hessian equation on a compact Kähler manifold $(X, \omega)$

$$
(\omega+i \partial \bar{\partial} u)^{k} \wedge \omega^{n-k}=f(z) \omega^{n}
$$

with $0 \leq f \in L^{p}(X)$ satisfying the normalization condition $\int_{X} f \omega^{n}=\int_{X} \omega^{n}$. What is the best possible regularity one can expect for the solution $u$ ?

We can also ask similar question with the condition on $f$ being replaced by $f \in C^{0, \delta}$ for some $0<\delta<1$. Indeed, by varying the parameters $a$ and $b$ in the example provided in Lemma 4.4, we have some assertions about what kind of regularity one can expect under different conditions of the right hand side function.

Example 4.6 (1) Let $b=-\frac{2 a}{p}+2$ with $p>1$. Then $f \in L^{p}$. (In fact, any $b>2 a / p-$ $2(n-1) / p$ yields $L^{p}$ right hand side). But in (4.10)

$$
\theta=\frac{2}{k}\left(1-\frac{1}{p}\right)+2 \frac{1}{a}=\frac{2}{k}\left(1-\frac{1}{p}\right)+O\left(\frac{1}{a}\right) \text { as } a \rightarrow+\infty .
$$

This shows that we cannot get better than Hölder regularity for $\varphi$. Moreover, the Hölder exponent can be at most $\frac{2}{k}\left(1-\frac{1}{p}\right)$.

(2) Similarly, for $b=2$, we have $f \in \mathcal{C}^{0, \delta}$ for some small $\delta>0$ and

$$
\theta \leq \frac{2}{k}+\frac{2}{a}
$$


(3) For $k \geq 3$ and $b=(k-2)(a-2)-3$, we have $f^{\gamma} \in \mathcal{C}^{0,1}$ for $\gamma=\frac{a}{(k-2)(a-1)-3} \rightarrow \frac{1}{k-2}$ as $a \rightarrow+\infty$. We can compute

$$
\theta=1-\frac{1}{a k}
$$

(4) For $b=2(k-1)(a-2)$, we have $f^{\gamma} \in \mathcal{C}^{1,1}$ for $\gamma=\frac{1+2 /(a-2)}{k-1} \rightarrow \frac{1}{k-1}$ as $a \rightarrow+\infty$. In this case

$$
\theta=2-\frac{2(k-1)}{k a}<2 .
$$

To summarize, by varying the parameter $a$ and $b$, the examples imply

- For $p>1, \gamma>\frac{2}{k}\left(1-\frac{1}{p}\right): f \in L^{p} \nRightarrow \varphi \in \mathcal{C}^{0, \gamma}$.

- For $\gamma>0$ there is $\delta=\delta(n, \gamma)$ such that $f \in \mathcal{C}^{0, \delta} \nRightarrow \varphi \in \mathcal{C}^{1, \gamma}$.

- For $k \geq 3, \gamma>\frac{2}{k}$ there is $\delta=\delta(n, \gamma)$ such that $f \in \mathcal{C}^{0, \delta} \nRightarrow \varphi \in \mathcal{C}^{0, \gamma}$.

- For $k \geq 3, s>\frac{1}{k-2}$ there is $\gamma<1: f^{s} \in \mathcal{C}^{0,1} \nRightarrow \varphi \in \mathcal{C}^{0, \gamma}$.

- For $s>\frac{1}{k-1}$ there is $\gamma<1: f^{s} \in \mathcal{C}^{1,1} \nRightarrow \varphi \in \mathcal{C}^{1, \gamma}$.

\subsection{The case of complex Hessian equations in domains}

Finally we mention that in the case of the complex Hessian equation on domains the following examples can be constructed:

Example 4.7 Let $a, b \in \mathbb{R}$ be two numbers satisfying $0<b<2(k-1)(a-1)$. Consider the Dirichlet problem in the unit ball in $\mathbb{C}^{n}$

$$
\begin{cases}(i \partial \bar{\partial} u)^{k} \wedge \beta^{n-k}=F & \text { in } B \\ u=0 & \text { on } \partial B\end{cases}
$$

where the solution $u$ is assumed to be $k$-subharmonic and $F$ is given in (4.4). Then $u$ is not $\mathcal{C}^{1,1}$ in any neighbourhood of 0 . But, $F^{\gamma} \in \mathcal{C}^{1,1}(B)$ for any $\gamma>\frac{1}{k-1}+\frac{1}{(a-1)(k-1)}$. In particular, no exponent larger than $1 /(k-1)$ could yield $\mathcal{C}^{1,1}$ solutions in general.

Proof The proof repeats the previous cases once one establishes an analogue of Lemma 4.1. We leave the details to the Reader.

Acknowledgements This project was initiated when the first named author was visiting University of California at Irvine in the summer of 2016. He wishes to thank the Department of Mathematics for the warm hospitality.

Open Access This article is distributed under the terms of the Creative Commons Attribution 4.0 International License (http://creativecommons.org/licenses/by/4.0/), which permits unrestricted use, distribution, and reproduction in any medium, provided you give appropriate credit to the original author(s) and the source, provide a link to the Creative Commons license, and indicate if changes were made.

\section{References}

1. Błocki, Z.: Regularity of the degenerate Monge-Ampère equation on compact Kähler manifolds. Math. Z. 244, 153-161 (2003)

2. Błocki, Z.: Weak solutions to the complex Hessian equation. Ann. Inst. Fourier (Grenoble) 55(5), 17351756 (2005) 
3. Błocki, Z.: A gradient estimate in the Calabi-Yau theorem. Math. Ann. 344, 317-327 (2009)

4. Dinew, S., Lu, C.H.: Mixed Hessian inequalities and uniqueness in the class $E(X, \omega, m)$. Math. Z. 279(34), 753-766 (2015)

5. Dinew, S., Kołodziej, S.: A priori estimates for complex Hessian equations. Anal. PDE 7(1), 227-244 (2014)

6. Dinew, S., Kołodziej, S.: Liouville and Calabi-Yau type theorems for complex Hessian equations. Am. J. Math. 139, 403-415 (2017)

7. Guan, P.: Extremal function associated to intrinsic norms. Ann. Math. 156, 197-211 (2002)

8. Guan, P., Trudinger, N., Wang, X.-J.: On the Dirichlet problem for degenerate Monge-Ampère equations. Acta Math. 182(1), 87-104 (1999)

9. Hou, Z.: Complex Hessian equation on Kähler manifold. Int. Math. Res. Not. 16, 3098-3111 (2009)

10. Hou, Z., Ma, X.-N., Wu, D.: A second order estimate for complex Hessian equations on a compact Kähler manifold. Math. Res. Lett. 17, 547-561 (2010)

11. Ivochkina, N., Trudinger, N., Wang, X.-J.: The Dirichlet problem for degenerate Hessian equations. Commun. Part. Differ. Equ. 29, 219-235 (2004)

12. Krylov, N.V.: Smoothness of the payoff function for a controllable process in a domain. Math. USSR-Izv. 34, 65-95 (1990)

13. Krylov, N.V.: Weak interior second order derivative estimates for degenerate nonlinear elliptic equations. Differ. Integr. Equ. 7, 133-156 (1994)

14. Li, S.-Y.: On the Dirichlet problems for symmetric function equations of the eigenvalues of the complex Hessian. Asian J. Math. 8, 87-106 (2004)

15. Lu, C.H.: Solutions to degenerate complex Hessian equations. J. Math. Pures Appl. 100, 785-805 (2013)

16. Phong, D.H., Picard, S., Zhang, X.: The Fu-Yau equation with negative slope parameter. Invent. Math. 209(2), 541-576 (2017)

17. Phong, D.H., Picard, S., Zhang, X.: Fu-Yau Hessian equation. J. Differ. Geom. (to appear). arXiv: 1801.09842

18. Phong, D.H., Tô, D.T.: Fully nonlinear parabolic equations on compact Hermitian manifolds. arXiv: 1711.10697

19. Pliś, S.: A counterexample to the regularity of the degenerate complex Monge-Ampère equation. Ann. Polon. Math. 86(2), 171-175 (2005)

20. Szekelyhidi, G.: Fully non-linear elliptic equations on compact Hermitian manifolds. J. Differ. Geom. 109(2), 337-378 (2018)

21. Tosatti, V., Weinkove, B.: The Monge-Ampère equation for $(n-1)$-pluri-subharmonic functions on a compact Kähler manifold. J. Am. Math. Soc. 30(2), 311-346 (2017)

22. Wang, Q., Xu, C.-J.: $C^{1,1}$ solution of the Dirichlet problem for degenerate k-Hessian equations. Nonlinear Anal. 104, 133-146 (2014)

23. Wang, X.-J.: Some counterexamples to the regularity of Monge-Ampère equations. Proc. Am. Math. Soc. 123, 841-845 (1995)

24. Wang, X.-J.: The k-Hessian equation. In: Geometric Analysis and PDEs, Lecture Notes in Math., 1977, pp. 177-252. Springer, Dordrecht (2009)

25. Yau, S.T.: On the Ricci curvature of a compact Kähler manifold and the complex Monge-Ampere equation. Commun. Pure Appl. Math. 31, 339-411 (1978)

Publisher's Note Springer Nature remains neutral with regard to jurisdictional claims in published maps and institutional affiliations. 